\title{
Estudio comparativo del patrocinio de equipos profesionales en España. El caso del futbol, baloncesto, balonmanoy hockey Comparative study of professional teams sponsorships in Spain. The case of football, basketball, handball, and hockey \\ Joan Borrisser Roldán, Francesc Solanellas Donato \\ Universidad de Barcelona (España)
}

Resumen. Este artículo tiene como objetivo estudiar el patrocinio deportivo en España basándose en los deportes de futbol, baloncesto, balonmanoy hockey patines. A pesar del contexto socio-económico del país, el patrocinio deportivo es una de las herramientas más utilizadas para generar ingresos. Los deportes mencionados son los deportes de equipo más practicados en nuestro país y utilizan el patrocinio como herramienta para obtener más recursos que les permitan conseguir sus objetivos. Sin embargo, nuestra hipótesis es que existen diferencias en la manera cómo utilizan el patrocinio los diferentes deportes. El número de patrocinadores, el tipo de patrocinio, su visibilidad, su presencia en la página web son algunas de las variables utilizadas para realizar dicha comparación. Los resultados confirman la hipótesis que existen diferencias significativas entre los diferentes deportes especialmente por lo que hace referencia al número de patrocinadores y como emplean los naming right. Consideramos que los datos que se aportan en este estudio son un primer paso en el desarrollo de la metodología de análisis del patrocinio. Este hecho puede permitirnos ampliar el estudio a más deportes, más organizaciones deportivas y a más territorios lo que nos permitiría avanzar en un estudio más global sobre el patrocinio deportivo.

Palabras clave. Patrocinio deportivo, deportes colectivos, ingresos, marketing relacional y notoriedad.

Abstract. The aim of this paper is to study the sport sponsorships in Spain based on team sports such as basketball, handball, football, and roller hockey. Although the Spanish socio-economic context is not good, sponsorships are among the most common tools to generate revenues. The referred team sports are the most practiced in Spain and they are using sponsorship to achieve their yearly objectives. However, our hypothesis is that there are differences about how each of the analyzed sport federation is using their sponsors. The number of sponsors, type of sponsorship, and visibility in the web page are some of the variables used to compare among sports. Results confirm our hypothesis that there are significant differences among these sports. We should underline variables like the number of sponsors by club and how they use the naming rights. This paper should be the first step in order to develop a methodology to analyze sponsorship. Consequently, we can extend our research to other sports and countries. Doing so, this will allow us to progress in a more global view of Sponsorship over the world.

Key words. Sports Sponsorship, team sports, revenues, relationship marketing, awareness.

\section{Introducción}

En las últimas décadas, el patrocinio deportivo ha crecido en el deporte profesional de nuestro país. La necesidad de clubs, federaciones y otras organizaciones deportivas de generar ingresos ha dinamizado el mercado. Este hecho, se ha visto favorecido por la crisis económica que ha reducido el poder adquisitivo de los socios y de los propios clubs.

Para Slack \& Barret (1999), antes de los años 70 el patrocinio era básicamente una actividad filantrópica. En España viene practicándose la esponsorización deportiva con carácter más o menos formal desde principios de los años ochenta (Bello, 1989).

Por ello, la gestión del patrocinio en las últimas décadas ha sufrido un cambio importante. Esto ha provocado que el patrocinio mediante la innovación, la necesidad y la originalidad los clubs inventen nuevos activos que provoquen más inversión en patrocinio. De acuerdo con Gómez (1993), las empresas aportaron más en patrocinio y esponsorización deportiva a partir de los Juegos Olímpicos de Barcelona 92. Se podría decir que fue el punto de inflexión para el desarrollo del patrocinio deportivo en España.

Históricamente, el concepto actual de sponsoring viene deEE.UU, donde nació en la segunda mitad del siglo XIX, refiriéndose a la subvención de las universidades a sus equipos de béisbol (Antoine, 2007).

En España, para designar a la persona que presta ayuda económica para la realización de actividades deportivas, benéficas, culturales, científicas, etc., en contrapartida a la colaboración publicitaria que el beneficiario de la ayuda debe prestar, se suelen utilizar diversos vocablos: «sponson», «mecenas», «filántropo» y «patrocinador» (Antoine, 2007). En las versiones antiguas del Diccionario de la Real Academia Española, el patrocinio se identificaba con «amparo, protección, auxilio»; y la voz patrocinador con «defender, proteger, amparar y favorecer». Sin em-

Fecha recepción: 22-07-17. Fecha de aceptación: 16-01-18

Joan Borrisser Roldán

borrisser@hotmail.com bargo la edición (2001) contiene ya las voces esponsorización y esponsorizar, a este última se relaciona con "patrocinar», entendido como «sufragar gastos con fines publicitarios» (Diccionario Real Academia Española, 2001). Por tanto, esponsorizar y patrocinar actuarán como sinónimos para apoyar o financiar una actividad.

Como dice Gómez, dos de cada tres empresas opina que la esponsorización y el patrocinio deportivo es fundamentalmente un modo de comunicación (Gómez, 2001).

Plan de patrocinio es el conjunto de acciones emprendidas por el patrocinador con el fin de obtener un mayor rendimiento informativocomercial como consecuencia de su colaboración, al margen de aquellos beneficios ya garantizados por su adscripción al programa (Maragas, Carroggio, Jones, Gutiérrez \& Garcia, 2003).

Para Campos (1997), el patrocinio deportivo es como el marketing para promover la venta a las empresas de los valores comunicativos que el deporte puede transmitir. Las empresas han considerado al patrocinio como una opción en su plan de comunicación adoptándola como una herramienta de comunicación (Campos, 1997). Femenía (2012) sitúa el volumen global en España en cerca de los 540 millones de euros. Es por este motivo que la potenciación del patrocinio es una realidad y hay que analizar la situación actual para explotarla más y tener unos beneficios mayores tanto para los clubs como para las empresas.

Asimismo y en la misma dirección, los cambios en la legislación publicitaria y la actitud cada vez más crítica del consumidor con la publicidad tradicional, ha permitido en los últimos años un crecimiento de la esponsorización y del patrocinio deportivo (Davis, 1994)(Desbordes, Ohl \& Tribou, 2001).

Gómez (2001) notó que hubo un relativo aumento de empresas que se han decidido a esponsorizar deporte siguiendo una corriente general a la hora de buscar nuevas alternativas de comunicación comercial.

Las actividades de patrocinio y mecenazgo son, indudablemente, una actividad de marketing con gran auge en los últimos tiempos, pero cuya efectividad y rendimiento presentan grandes dificultades de medición (Calderón, Nicolau, \& Mas, 2003). 
El mercado se ha apoderado del futbol por una sociedad de masas y de consumo, donde las cadenas televisivas se hacen con los derechos de retransmisión y las casas comerciales compran derechos de imagen de los jugadores, todo ello con fines mercantiles (Acuña \& Acuña, 2018). Urriolagoitia (2007) explicaba que las compañías que consideraron sus acuerdos de patrocinio como un recurso estratégico, alcanzaron eléxito.

En el estudio presentado por Alonso-Dos-Santos, Calabuig, Montoro \& Valantine (2017) se confirma que la imagen del patrocinado influye en la imagen del patrocinador y a su vez en la intención de compra de los productos, siendo el patrocinio efectivo.

Tal como nombra Pérez-González, García-Unanue, SánchezSánchez, Sánchez-Burón \& Burillo (2018) la demanda de productos deportivos se basa en ropa y equipamiento, cuya finalidad es tanto la práctica deportiva como la de seguir las tendencias de moda, ambos aspectos con mucho peso en la decisión de compra.

Si bien todo parece apuntar que el patrocinio deportivo se ha dinamizado en los últimos años y hay una mayor presencia de las empresas en los equipos profesionales, no se han planteado estudios que nos permitan conocer cómo ha evolucionado exactamente el número de patrocinadores de los diferentes equipos profesionales en España y que diferencias existen entre los diferentes deportes.

El estudio realizado ha ido centrado en los deportes de equipo masculinos pero cabe destacar que de acuerdo con Leruite, Cabrera, \& Zabala (2015), las ayudas concedidas por las administraciones públicas y las becas $\mathrm{ADO}$ son muy precarias para los deportes femeninos y para la visibilidad de los mismos.

La investigación que se presenta a continuación plantea conocer la situación del patrocinio de los principales deportes profesionales en España. Este estudio inicial pretende al mismo tiempo, presentar la metodología para poder en un futuro abordar otros deportes y otras organizaciones deportivas de diferentes países.

Por consiguiente, en esta línea, la finalidad del estudio es comparar el patrocinio actual de los equipos profesionales de primera y segunda división españolas en los deportes del futbol, baloncesto, balonmano y hockey patines, debido a que estos son los deportes colectivos con más seguidores y practicantes en Cataluña («Hàbits Esportius a Barcelona - 2013,» 2014). Para ello el estudio se estructura de la siguiente forma. En primer lugar, se aborda el diseño de la investigación, especificando la metodología de análisis empleada, así como el proceso de recogida de datos dividiéndose en tres bloques. En segundo lugar, se exponen e interpretan los datos obtenidos y por último, se recogen las principales conclusiones.

\section{Material y método}

La investigación desarrollada se basa en la recopilación de datos de los clubs que conforman la primera y segunda división de futbol, baloncesto, balonmano y hockey que corresponden a la Liga Santander y 1,2,3, a la Liga Endesa y LEB ORO del baloncesto, a la Liga ASOBAL y División de Honor Plata del balonmano y a la OK Liga y Liga de Primera División Nacional del Hockey sobre Patines.

\begin{tabular}{lccc}
$\begin{array}{l}\text { Tabla 1. } \\
\text { Muestra del Estudio }\end{array}$ & & & \\
\hline Deportes & Total & Equipos 1 ${ }^{\text {a división }}$ & Equipos 2 $2^{\text {a división }}$ \\
\hline Baloncesto & 34 & 18 & 16 \\
Balonmano & 31 & 15 & 16 \\
Futbol & 42 & 20 & 22 \\
Hockey & 30 & 16 & 14 \\
\hline
\end{tabular}

\section{Muestra del estudio}

El universo del estudio serían todos los clubs y deportes de España pero nos hemos centrado en extraer los datos de los 137 clubs que pertenecen a dichos deportes y categorías. La selección de la muestra se concreta con los cuatro deportes colectivos con más seguidores y practicantes en Cataluña («Hàbits Esportius a Barcelona - 2013,» 2014)

La muestra por tanto se ha dividido en 42 equipos de futbol, $34 \mathrm{de}$ baloncesto, 31 de balonmano y 30 de hockey sobre patines.

La información analizada se ha obtenido mediante las páginas web oficiales de los equipos comentados con anterioridad. Para ello, se creó una ficha de recogida de datos para recoger la misma información de los distintos clubs y se procedió a realizar una segunda revisión de todos los datos recogidos. Esta ficha servirá para apoyar otros estudios con una temática similar

Toda la información que se ha extraído de los clubs es la siguiente:

Bloque 1. Contiene las categorías de patrocinio existentes, el total de patrocinadores y los naming right.

- Número de categorías. Tanto los clubs como las empresas tienen una jerarquía en sus patrocinadores. Por tanto, esta sección hace referencia a la cantidad de categorías que tienen en los deportes, como por ejemplo, patrocinador principal, patrocinador secundario y colaborador. En este caso, el número de categorías sería tres.

- Total de patrocinadores. El total de patrocinadores sirve para conocer la media de las categorías y deportes. En esta sección se ha contabilizado todas las empresas que aparecen en la página web.

- Naming rights. El naming right se puede traducir por «derecho de nombre o «derecho para nombrar». Consiste en un modo de patrocinio en el que una empresa - patrocinador adquiere los derechos para poner el nombre de la marca a equipo, una competición, un estadio o una instalación (Femenía, 2012). Este activo tan explotado en algunas competiciones llegó a España con la intención de dar nombre a los estadios o clubs siendo una fuente muy potente de patrocinio pero aun sin consolidarse.

Bloque 2. Contiene el Banner, el tipo de dominio y los clics para acceder la información recogida directamente desde la página oficial de los clubs.

- Banner. El Banner se refiere a la lengüeta o pestaña que aparece en las distintas páginas web o en un apartado de patrocinadores. En este caso, se ha contabilizado como banner directamente cualquier etiqueta que apareciera en la página principal, es decir, cualquier palabra referente a los partners, como pueden ser colaboradores, patrocinadores, club de empresas, sponsors, etc.

- Dominio de la página web. La recopilación de este apartado se ha enfocado para conocer que dominio usan los equipos en función de la categoría y deporte, es decir, si tienen «.com, .es, .cat, etc» para así situar el patrocinio en un carácter más local, nacional o internacional en función de los clubs.

- Cantidad de pasos para llegar a los patrocinadores. Son los clicks que tienes que «clicar» para llegar a la página o apartado de patrocinadores. En el caso que no hubiera sección para ellos y aparecieran en la parte inferior de la página web o en cualquier otra zona, se ha cogido el criterio de las bajadas con la barra espaciadora.

Bloque 3. Contiene la dimensión empresarial comparando el análisis con los sectores de Sanajahu, Campos, Breva \& Mut (2015) y los sectores de los patrocinadores principales.

- Sector empresarial. Como se ha visto en el trabajo realizado por Sanajahu et al. (2015), ellos cogieron 7 sectores para su división de sectores empresariales en función de los resultados de las Pymes que tuvieron (alimentación, finanzas, automoción, distribución, textil, energía y otros) (Sanajahu, et al., 2015, p. 11). Se ha considerado oportuno comparar los resultados con este artículo para observar las diferencias obtenidas con estos mismos sectores empresariales. Para apreciar la información, las bebidas se han incluido en el sector alimentario y en el apartado de «otros» se han incorporado todos los patrocinadores que no se podían adjuntar en estos sectores.

La tendencia del patrocinio deportivo está indiscutiblemente en alza (Antoine, 2007). Es por este motivo que se cree que estas variables ayudarán a analizar la situación actual del patrocinio en el deporte español.

El análisis estadístico se ha realizado mediante el Programa «SPSS Statistics» la versión 18 y Excel 2013.

Las distintas pruebas que se ha usado para analizar el contenido han sido la mediana, la moda, frecuencias, chi cuadrado, test ANOVAy test de TUKEY. Estos test nos han servido para conocer y comparar los 
distintos deportes, ver las diferencias que existen entre ellos en función de nuestras variables y las tendencias que aparecen. El test Anova ha servido para conocer las diferencias de las distintas medias entre clubs y por último se ha aplicado el test de Tukey para ver las diferencias significativas entre deportes y hacer una comparación conjunta. Con ello se ha valorado si la $p$ valor de la prueba chi cuadrado es mayor o menor de .05\% para conocer si se rechaza o se aprueba que hay diferencias significativas entre deportes.

\section{Resultados}

Los resultados del estudio se han dividido en los tres bloques de análisis.

Todos estos resultados irán relacionados con los distintos deportes y categorías para conocer las diferencias y similitudes que existen entre ellos y contrastar así la hipótesis planteada.

\section{Bloque 1.}

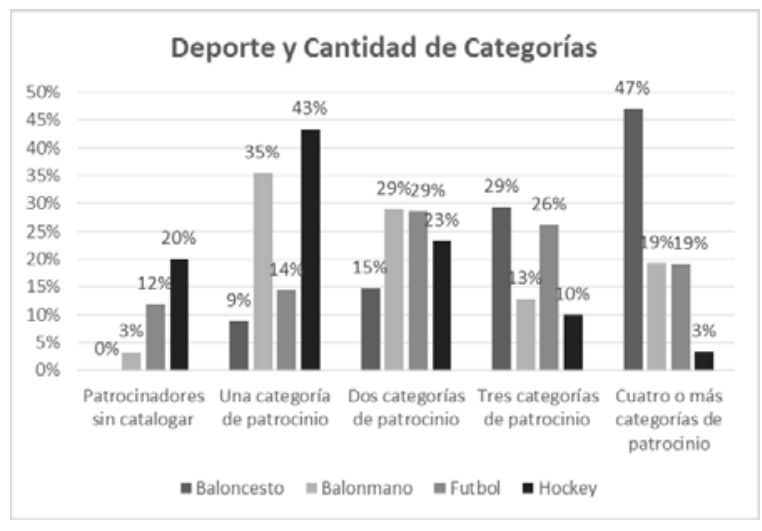

Figura 1. Gráfico comparativo entre los deportes y cantidad de categorías

El $p$ valor de la prueba chi-cuadrado en relación entre el deporte y la cantidad de categorías es de .000 demostrando que son variables dependientes, es decir, existe una relación entre ellas.

El deportequemenos categoriza a sus colaboradores opatrocinadores con un $20 \%$ de los equipos, es el hockey patines. Sin embargo, se observa que este deporte engloba a sus partners en una única categoría de patrocinio, con un $43 \%$ siendo su resultado más destacable.

Es necesario realzar que el baloncesto es el equipo que usa más de cuatro categorías para fraccionar a sus patrocinadores en un $47 \%$ de los clubs y tiende hacia una línea ascendente a categorizar más. Además, es el deporte donde no posee ningún patrocinador sin categorizar.

Por otro lado el futbol, no tiene unas diferencias destacables entre dos y tres categorías de patrocinio manteniéndose entre un 29\% y 26\% siendo el deporte con la repartición más equitativa entre estas categorías.

Por último, en el balonmano se puede observar que un $3 \%$ de sus clubs no tiene ninguna categorización, un 35\% una, 29\% dos, un 13\% tres y un $19 \%$ más de cuatro categorías. Es el segundo deporte que tiene

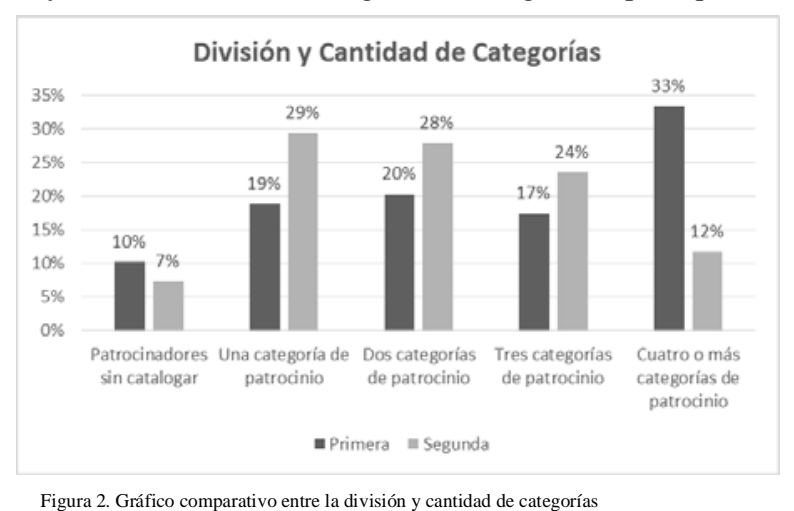

más de cuatro categorizaciones para dividir a sus patrocinadores junto al futbol.

En la figura 2 que pertenece a la relación entre la división y la cantidad de categorías se ha observado en la prueba de chi cuadrado que hay diferencias significativas. El resultado de dicha prueba ha sido .034 donde se presenta que en primera división un 33\% de los equipos tienen cuatro o más categorías de patrocinio. Por el contrario, en segunda división se observa que los resultados van de forma descendente, donde un $29 \%$ de los equipos tienen tan solo una categoría de patrocinador.

Cabe destacar que hay más equipos sin categorizar en primera división, que en segunda con un $10 \%$ y $7 \%$ respectivamente.

Tabla 2 .

\begin{tabular}{|c|c|c|c|c|c|c|c|c|}
\hline \\
\hline & \multirow{2}{*}{$\mathrm{N}$} & \multirow{2}{*}{ Media } & \multirow{2}{*}{$\begin{array}{c}\text { Desviación } \\
\text { típica }\end{array}$} & \multirow{2}{*}{ Error típico } & \multicolumn{2}{|c|}{$\begin{array}{l}\text { Intervalo de confianza } \\
\text { para la media al } 95 \%\end{array}$} & \multirow{2}{*}{-Mínimo } & \multirow{2}{*}{ Máximo } \\
\hline & & & & & $\begin{array}{l}\text { Límite } \\
\text { inferior }\end{array}$ & $\begin{array}{c}\text { Límite } \\
\text { superior }\end{array}$ & & \\
\hline Futbol & 42 & 13.93 & 9.809 & 1.514 & 10.87 & 16.99 & 3 & 47 \\
\hline Baloncesto & 34 & 38.06 & 25.071 & 4.300 & 29.31 & 46.81 & 3 & 95 \\
\hline Balonmano & 31 & 23.94 & 25.441 & 4.569 & 14.60 & 33.27 & 2 & 119 \\
\hline Hockey & 30 & 12.83 & 11.786 & 2.152 & 8.43 & 17.23 & 0 & 50 \\
\hline Total & 137 & 21.94 & 21.398 & 1.828 & 18.33 & 25.56 & 0 & 119 \\
\hline
\end{tabular}

Como se percibe en la tabla 2, la media de patrocinadores es bastante dispar en función del deporte. En el futbol la media de patrocinadores por equipo es de 14, en el baloncesto 38, en el balonmano 24 y por último en el hockey es de 13 patrocinadores.

A nivel español la media en estos cuatro deportes podemos comprobar que es de 22 patrocinadores.

Para demostrar que el número medio de patrocinadores varía de forma general en función del deporte se ha realizado el Test Anova siendo el $p$ valor .000 rechazando la hipótesis nula de igualdad de medias y corroborando la hipótesis planteada.

Para profundizar más y encontrar las diferencias en función de cada uno de los deportes también se ha desarrollado la prueba de Tukey para realizar una comparación múltiple por deportes de las medias de patrocinadores.

Tabla

\begin{tabular}{|c|c|c|c|c|c|}
\hline & \multirow{2}{*}{ Deporte } & \multirow{2}{*}{ Deporte } & \multirow{2}{*}{ Sig. } & \multicolumn{2}{|c|}{ Intervalo de confianza al 95\% } \\
\hline & & & & Límite inferior & Límite superior \\
\hline \multirow{12}{*}{ Dimensión } & \multirow{3}{*}{ Futbol } & Baloncesto & .000 & -35.55 & -12.71 \\
\hline & & Balonmano & .123 & -21.73 & 1.71 \\
\hline & & Hockey & .995 & -10.74 & 12.93 \\
\hline & \multirow{3}{*}{ Baloncesto } & Futbol & .000 & 12.71 & 35.55 \\
\hline & & Balonmano & .017 & 1.83 & 26.41 \\
\hline & & Hockey & .000 & 12.83 & 37.62 \\
\hline & \multirow{3}{*}{ Balonmano } & Futbol & .123 & -1.71 & 21.73 \\
\hline & & Baloncesto & .017 & -26.41 & -1.83 \\
\hline & & Hockey & .108 & -1.57 & 23.78 \\
\hline & \multirow{3}{*}{ Hockey } & Futbol & .995 & -12.93 & 10.74 \\
\hline & & Baloncesto & .000 & $\begin{array}{l}-12.50 \\
-37.62\end{array}$ & -12.83 \\
\hline & & Balonmano & .108 & -23.78 & 1.57 \\
\hline
\end{tabular}

Como se analiza en la tabla 3, hay diferencias significativas con el baloncesto respecto a los otros deportes. En cambio, no hay diferencias significativas entre el futbol, el balonmano y el hockey.

Tabla 4.

División y mediana de patrocinadores

\begin{tabular}{ccccc}
\multicolumn{2}{l}{ División y mediana de patrocinadores } & & & \\
\hline División & $\mathrm{N}$ & Media & \multicolumn{2}{l}{ Intervalo de confianza para la media al 95\% } \\
\cline { 4 - 5 } & 69 & 24.46 & Límite inferior & Límite superior \\
\hline Primera & 68 & 19.38 & 19.18 & 29.75 \\
Segunda & 137 & 21.94 & 14.39 & 24.38 \\
Total & & & & \\
\hline
\end{tabular}

Por otro lado, haciendo referencia a primera y segunda división podemos comprobar en la tabla 4, que la media total de patrocinadores en las máximas categorías es de 24 y en segunda división es de 19. El resultado del test Anova fue .165 demostrando que no hay diferencias significativas entre todos los equipos de primera y segunda división analizados.

En la figura 3 se puede contemplar que en el futbol y el hockey aun no se ha consolidado este activo de patrocinio. Por el contrario, el baloncesto y el balonmano son dos deportes que usan más el naming right en sus clubs con más de la mitad de los equipos con un activo de este tipo.

En la figura 4, se puede analizar que tanto en primera como en segunda división más de la mitad de los equipos no usa los naming right como fuente de ingreso. 


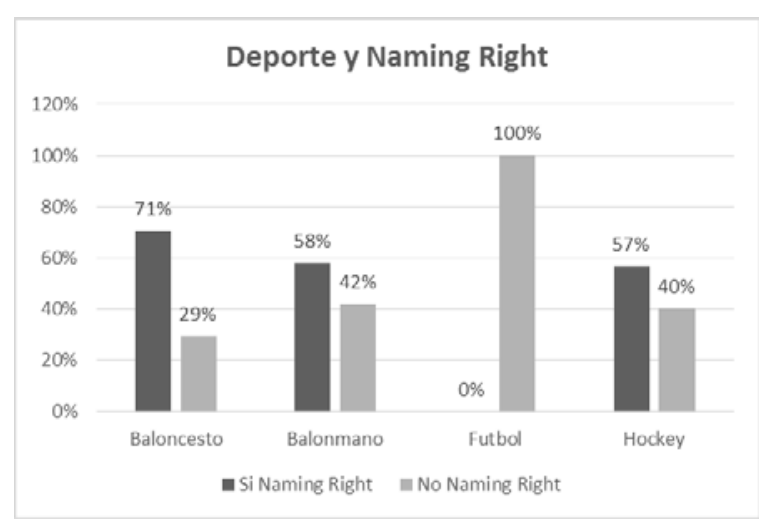

Figura 3. Gráfico comparativo entre deporte y naming right

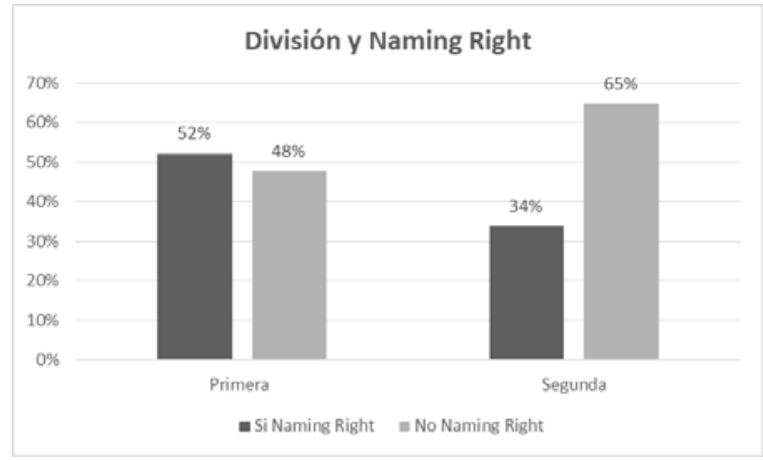

Figura 4. Gráfico comparativo entre división y naming right

Bloque 2.

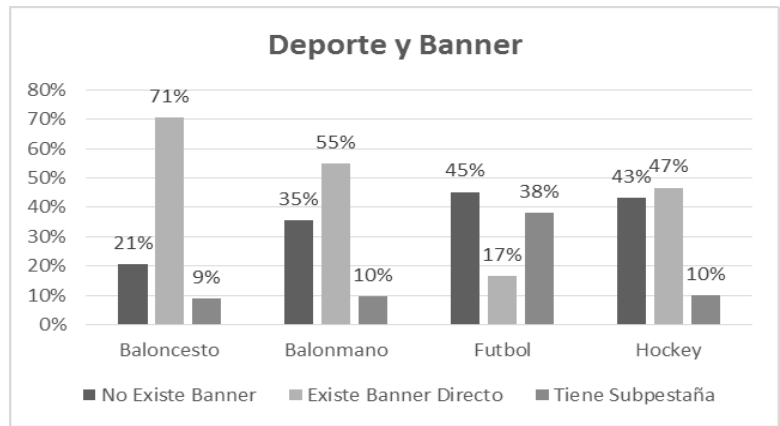

Figura 5. Gráfico comparativo de los deportes y banners.

Los resultados de la figura cinco relacionan el deporte y el banner de las páginas web mostrando que el baloncesto con un $71 \%$ de los equipos, es el deporte que demuestra más facilidad para localizar a los patrocinadores. Por otro lado, vemos que el futbol es el deporte donde los clubs no acostumbran a tener un banner directo pero si lo tienen en una sub-pestaña con un 38\%. En el balonmano se comprueba que tienen un apartado específico para sus empresas muy visible (55\%) y el hockey junto al futbol es el equipo que menos apartados específicos tienen en sus páginas web. Que no exista banner, no significa que no tengan patrocinadores, sino que no tienen un apartado específico en la página web de patrocinadores o colaboradores.

Los resultados de la comparativa entre deportes nos indican que no hay diferencias significativas ( $p \mathrm{~d}$ ».05) entre la primera división y la segunda división en referencia al tipo de banner que se utiliza para conectarse con los patrocinadores.

En la figura 6 que corresponde a la división y el banner, se ha observado que ( $p$ e» .953) es decir no existe una relación entre la división y el banner. Observando el gráfico se ve que los porcentajes son muy parejos entre ellos y que no hay grandes disimilitudes entre la división y los banners.
Como se puede observar en la figura 7 el dominio más predominante es «.com» seguido por «.es». El dominio «.cat» es destacado por el hockey patines, donde un $40 \%$ de los clubs llevan este dominio. Por otro lado, el dominio «.es» es usado por un 36\% de los equipos españoles de futbol. Lo más minoritarios son el «.eus», «.net», «.org» y «.ad».

Por otro lado, en la figura 8 se puede apreciar que un 64\% de los equipos de primera división de los deportes analizados usan el dominio «.com», seguido por «.es» $\mathrm{y}$ «.cat».

En segunda división los tres dominios más destacados son los mismos que en primera división. Los dominios de «.eus» y «.org» son más utilizados en segunda división y el «.net» es el menos usado junto $\mathrm{al} \ll$.ad».

En la figura 9, la $p$ valor de la prueba chi cuadrado es de .001 donde se rechaza la hipótesis nula de independencia, existiendo una relación los deportes y el número de clics.

Cabe destacar que había tres equipos considerados outliers que se han incluido en un clic y no tenían ningún tipo de patrocinio en sus equipos.

Se puede observar que el equipo con más dificultad para llegar a los patrocinadores es el futbol con más de 5 clics en un $17 \%$ de los equipos. Por otro lado, el balonmano en un $58 \%$ de los equipos, se consigue llegar con más facilidad para observar las empresas que colaboran con los

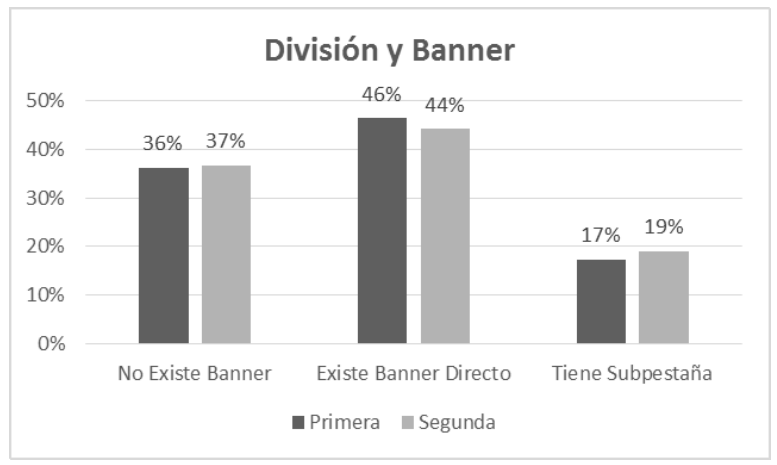

Figura 6. Gráfico comparativo entre división y banner

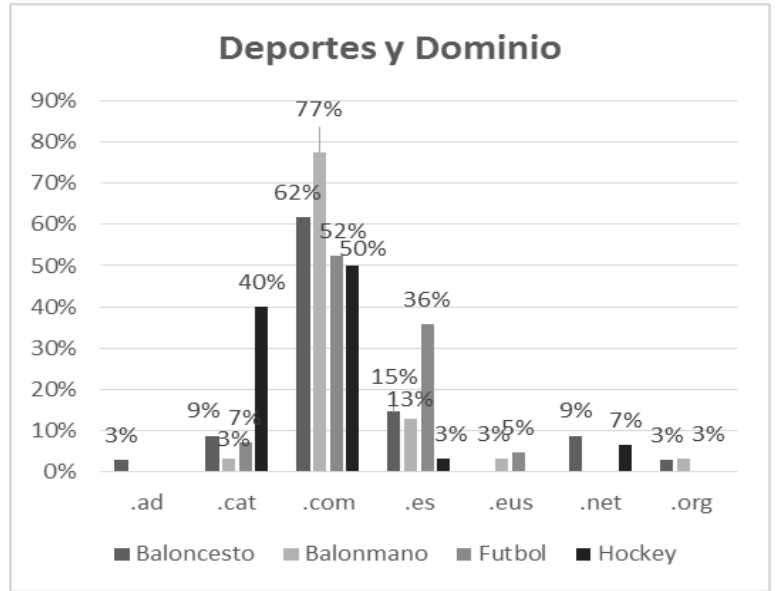

Figura 7. Gráfico comparativo entre deportes y dominio

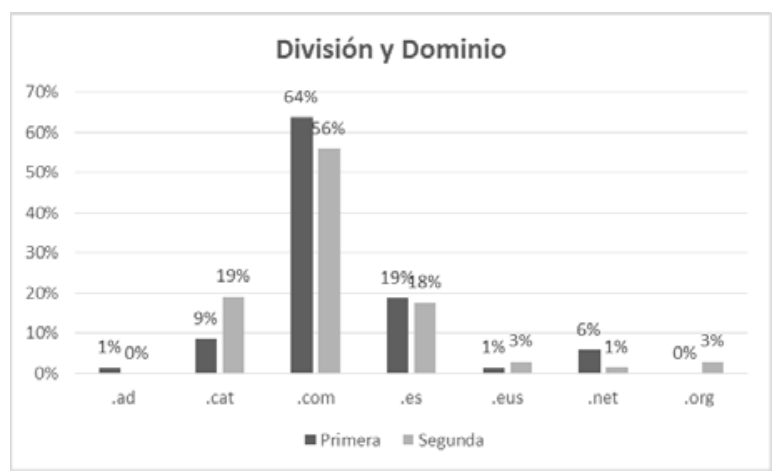

Figura 8. Gráfico comparativo entre división y dominio 


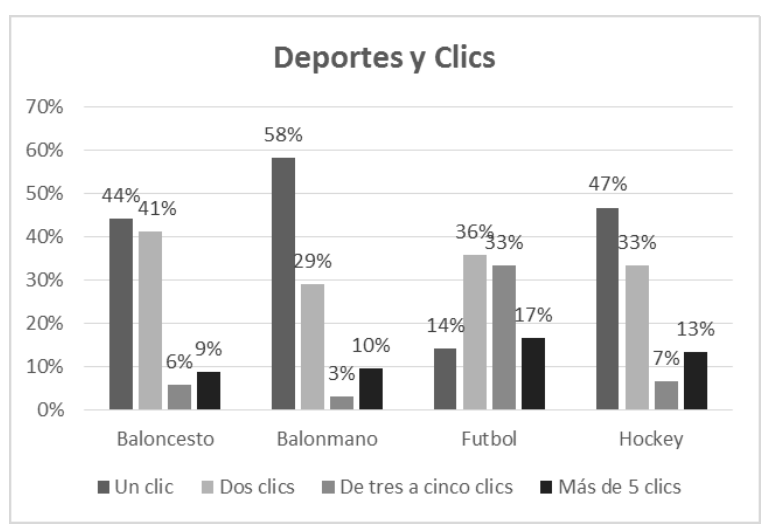

Figura 9. Gráfico comparativo entre deportes y clics

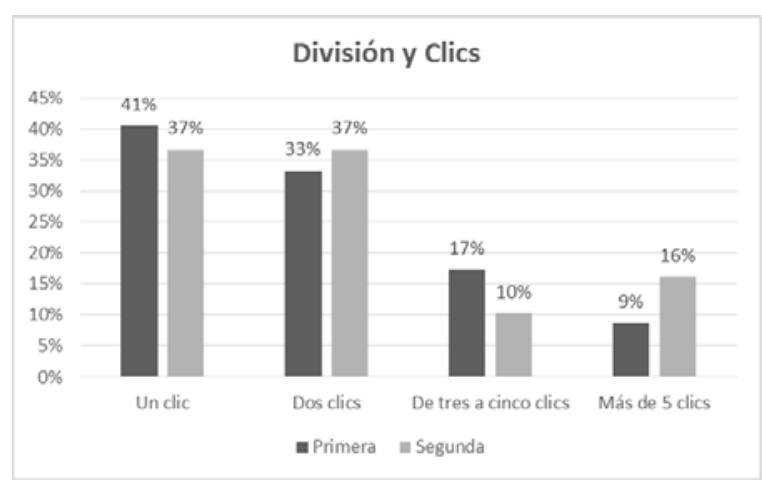

Figura 10. Gráfico comparativo entre división y clics

clubs seguidamente del hockey y el baloncesto. Con dos clics se observa que el baloncesto es el deporte que debe tener un sub apartado específico de patrocinadores con un 41\%, similar al futbol con un $36 \%$.

Es destacable la tercera columna de tres clics a cinco pasos para llegar a los patrocinadores, donde un 33\% de los equipos de futbol tienen que realizar un proceso más largo para llegar a sus patrocinadores.

En la figura 10 el $p$ valor de la prueba chi cuadrado es de .387 demostrando que las variables son independientes.

Se puede observar que primera y segunda división tienen unos datos muy parejos en lo referente a los primeros dos apartados. En el apartado de los steps o pasos para llegar a observar los patrocinadores lo más destacable es de tres a más de cinco clics, ya que hay un 26\% de los equipos analizados de primera división llegan a encontrar a los patrocinadores en esta categorización.

Se puede comprobar que ya es en segunda división donde más de un $16 \%$ de los equipos necesitan más de cinco clics para llegar al apartado de patrocinadores.

\section{Bloque 3.}

En este bloque se ha terminado de examinar los datos que se han recogido de los sectores empresariales y agrupar los patrocinadores principales más destacados en estos deportes y categorías.

Los sectores empresariales usados para la recogida de la muestra han sido extraídos del estudio sobre el estado del patrocinio en España (Sanajahu, et al., 2015).

Enel baloncesto, el sectormás destacado es con un21\% el alimentario, seguido por el financiero con un $18 \%$. Los sectores del balonmano son muy diversos ya que se recogen unos resultados similares entre los sectores de la alimentación, la automoción, el textil y el financiero. En el futbol las categorías empresariales más destacables son la alimentación, la financiación y la textil. Por último, en el hockey la más destacable es el sector alimentario, el textil y el financiero.

La figura 12 nos demuestra que en primera división los sectores más destacados son finanzas, textil y alimentación.

En segunda división el más destacado con un $24 \%$ es el sector alimenticio, seguido por el textil y financiero. El apartado de otros, ocurre lo mismo que en la figura anterior, ahí se engloban todas las empresas que no tienen categorización en estos sectores.

En la figura 13 se puede observar que los porcentajes que se han recogido en nuestro artículo están por debajo de los resultados de (Sanajahu, et al. 2015) exceptuando el textil y otros. La categorización de «otros» nos indica que hay muchas tipologías de empresas distintas a estos sectores, dando a entender que habría que ampliar la naturaleza de las distintas secciones.

En la figura 14 se puede observar, que el baloncesto busca

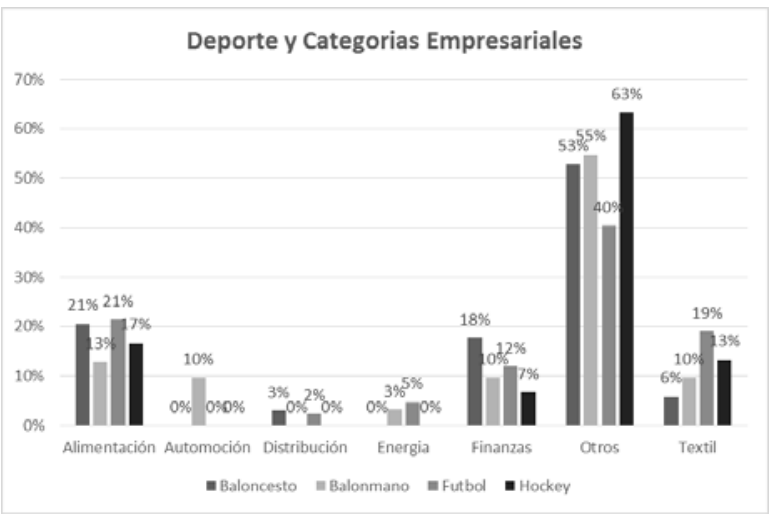

Figura 11. Gráfico comparativo entre deporte y categorías empresariales

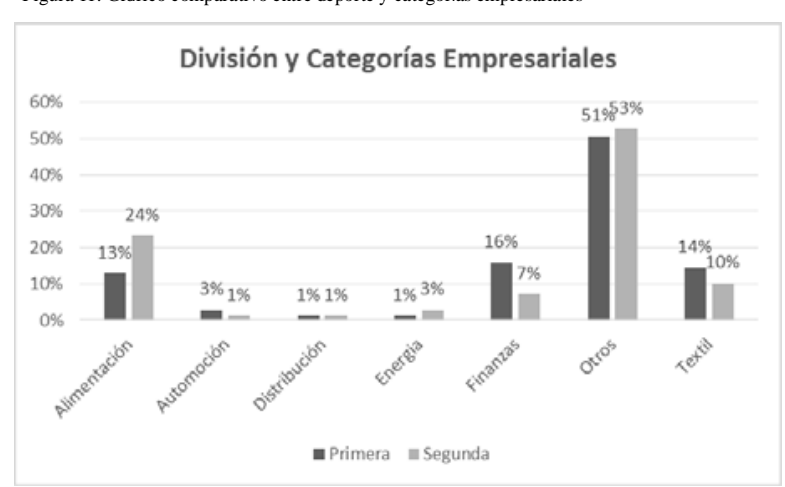

Figura 12. Gráfico comparativo entre división y categorías empresariales

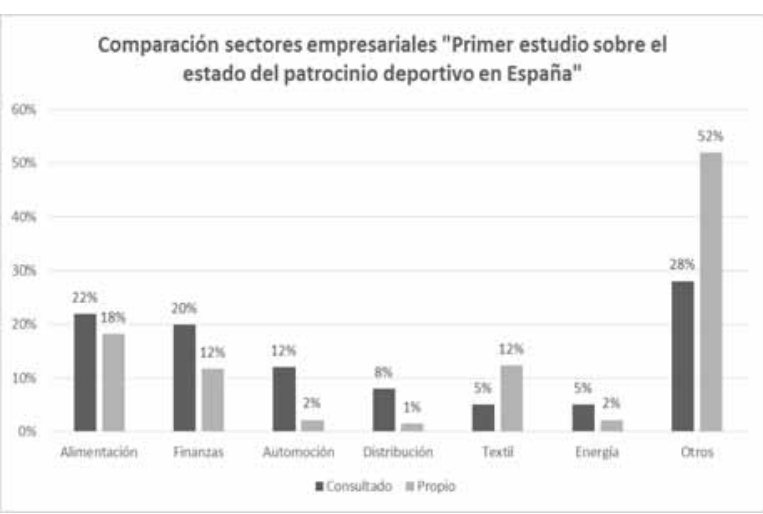

Figura 13. Gráfico comparativo entre los sectores empresariales propios y consultados

Relación de patrocinadores locales, nacionales y internacionales

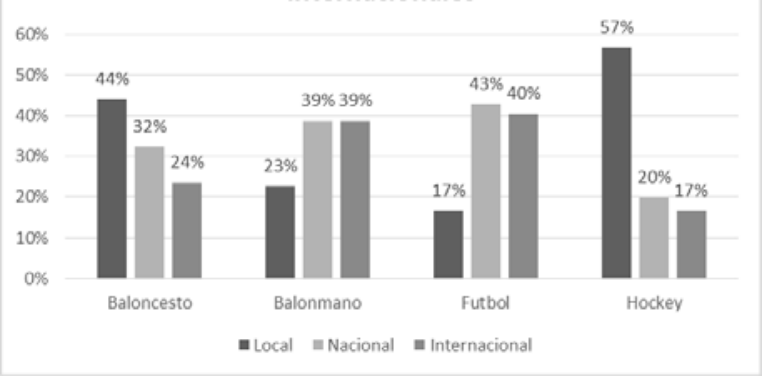

Figura 14. Gráfico comparativo entre deportes y patrocinios locales, nacionales y internacionales 
patrocinadores más locales, seguido por nacionales e internacionales. Por otro lado, el balonmano busca empresas más nacionales e internacionales para buscar así proyecciones más universales.

El futbol es el deporte que busca menos patrocinadores locales, justo al contrario que el hockey donde las empresas más locales son las fuentes mayoritarias de ingresos.

\section{Discusión}

\section{Bloque 1.}

Por este motivo, la captación de recursos se ha contabilizado en función de las categorías y de las medias de patrocinadores que existen en los distintos deportes. Se ha analizado que la tendencia en el futbol es obtener menos patrocinadores principales pero mucho más potentes que la de los otros deportes para obtener mayor exclusividad y visibilidad. Los resultados han demostrado que tener tres categorías es lo más apropiado para el desarrollo de sus clubs y para la visibilidad de ellos.

Siguiendo la línea de Acuña \& Acuña (2018), se ha podido observar que el futbol es el deporte que más impacto tiene respeto a los otros deportes. Los resultados han demostrado que tener tres categorías es lo más apropiado para el desarrollo de los clubs y para la propia visibilidad de ellos. Además siguiendo la visibilidad de patrocinadores en su investigación observamos que los patrocinares se hacen visibles desde el principio y en la cabecera, dónde la propia Liga en anteriores ediciones sellamaba Liga BBVA.

De acuerdo con Urriolagoitia (2007) para alcanzar el éxito mediante los recursos estratégicos, tiene que existir un equilibrio entre patrocinador y patrocinado adquiriendo tener una sinergia con los objetivos del club y empresa. Esta sinergia tal como ha presentado en su estudio Alonso-Dos-Santos et al. (2017) ha comprobado que la influencia de la imagen de la marca se relaciona con el deporte o evento que por consiguiente, hace que exista una relación o vinculación entre ellas.

De acuerdo con Pérez-González et al. (2018) en nuestro estudio se ha comprobado que las camisetas de los jugadores es un lugar muy apropiado para publicitar sus marcas.

El hockey en cambio busca englobar a todas sus colaboraciones en un mismo grupo de patrocinio para así obtener muchos ingresos de distintas empresas, debido a que este deporte no es tan común para conseguir un solo sponsor tan potente.

El balonmano prefiere tener una categoría de patrocinio marcando bien el patrocinador principal pero no le disgusta tener dos y tres categorías o hasta más de cuatro. Como dice Nogales (2006), en el balonmano no existe un trabajo profundo y profesional del patrocinio. Esto ayuda a entender que en función del deporte, la dificultad para gestionar y encontrar nuevos patrocinadores será más compleja. Es por ello que la categoría, los éxitos y los planes estratégicos de marketing y publicidad de los clubs deberían de estar bien enfocados para encontrar la eficacia del patrocinio deportivo.

El deporte que más resalta es el baloncesto, donde obtiene casi la mitad del patrocinio de todos los equipos, en cuatro o más categorías de patrocinio desarrollando muchas fracciones en sus colaboraciones. Esto nos da a entender que tienen muchas subcategorías para todas sus empresas siendo el deporte que más patrocinadores tiene de media.

El futbol tiene de promedio entre dos y tres categorías debido a que prefiere pocos colaboradores pero más poderosos. En primera división la jerarquización de los partners está más desarrollada debido a que tienen muchas empresas de diferentes tipos. Por el contrario, en segunda división una o dos categorías es lo más común en los deportes analizados. Probablemente porqué el interés de los patrocinadores en las categorías de segunda división es inferior. Sin embargo, es evidente que estos datos deberían corroborarse con los valores económicos de cada uno de ellos.

La claridad delos patrocinadores más destacable es la del balonmano, baloncesto y el hockey debido a que tienen muchas más colaboradores y son más propensos a abrir a nuevas líneas de patrocinio como los naming right. El futbol en cambio no ha sido tan partidario a presentar a los patrocinadores en primera línea en sus páginas web vendiendo el nombre de su club o estadio. Probablemente la repercusión social del futbol le impide en ocasiones a vender su nombre mientras que en otros deportes puede suponer la supervivencia del propio club. Como se ha visto en el estudio de (Uefa, 2015) los naming right, en España y Portugal aún no se han consolidado. Por consiguiente, la división en la que estén, siempre ayuda en estos tipos de activos y es por ello que en primera división es el lugar más idóneo para empezar a mostrar los naming right por la repercusión que existe en la máxima categoría.

\section{Bloque 2.}

En el segundo bloque se ha analizado la visibilidad mediante los banners, el dominio y los clics o steps para llegar a los patrocinadores.

Se ha observado que el deporte que más facilidad tiene para llegar a los patrocinadores es el baloncesto y el balonmano. Esto puede ser debido a que en estos dos deportes como se ha observado su propio nombre del equipo es el patrocinador. Por otro lado, el futbol es el deporte que más dificultad visual presenta en sus páginas web para sus partners, debido a que tienen una sub pestaña y además un 38\% de los equipos analizados no tienen banner pero si patrocinadores en la parte inferior de la página web. Aun así, el hecho de tener una sub pestaña puede ser positivo para la visibilidad de su página web. La diferencia entre división y banner se ha comprobado que es mínima. La mayoría de los equipos de primera división tienen un banner directo o sub pestaña debido a la profesionalización del sector. Cabe destacar que un 36\% y $37 \%$ de los clubs no tienen banner en sus páginas web y puede ser debido a que tengan algún otro canal de comunicación sobre sus patrocinadores.

El deporte que tiene un dominio más local es el hockey patines. Esto significa que sus patrocinadores serán de un entorno más cercano y/o familiar. También es debido a que el hockey no tiene tanta repercusión como otros deportes y es por ello que los patrocinadores no serán habitualmente multinacionales. En cambio el futbol junto al baloncesto y al balonmano donde el dominio es «.com», buscan más la internacionalidad en sus posibles sponsors. En primera división se usan más los dominios «.com y .es». Por el contrario, en segunda división el dominio local «.cat» coge la misma fuerza que el dominio nacional.

La visibilidad de un patrocinador en las dos máximas categorías se ha comprobado que es bastante eficaz ya que con tan solo dos clics se consigue observar a los patrocinadores en la mayoría de clubs.

\section{Bloque 3.}

Los sectores empresariales más potentes son los relacionados con la alimentación, el financiero y el textil. La ayuda económica que reciben los clubs de futbol y baloncesto de los bancos es muy importante, debido a que son una fuente considerable a tener en cuenta para conseguir esas cifras en sus facturaciones. El sector alimentario, donde se han incluido las bebidas (mayoritariamente cervezas), es un punto clave en los distintos deportes, debido a la relación con el público que observa estos partidos. El sponsor principal en muchos clubs se ha comprobado que es del sector alimentario seguido por el textil. Los proveedores de material deportivo de los clubs son muy importantes en los ingresos por patrocinio y es por este motivo que en muchas ocasiones son los patrocinadores principales. Otros sectores como la energía, la automoción y la distribuciónno destacan tanto en estos deportes como patrocinadores principales. Sin embargo, es posible que las tendencias en un futuro vayan cambiando ofreciendo otros sectores en el deporte. Como se vio en Carroggio (1994), los sectores empresariales eran totalmente distintos y no había ningún partner relacionado con la energía y muchos clubs inclusive aun no tenían ni patrocinadores oficiales. Por esta razón, es posible que a nivel legislativo o a nivel comunitario, los patrocinadores puedan ir cambiando hacia un carácter más social, energético o cualquier otro sector.

Antiguamente el tabaco empezó a ser una fuente principal de ingresos en muchos deportes pero la legislación evitó que se publicitara este adictivo. La cerveza es uno de los patrocinadores más importantes a nivel de ingresos y si la ley prohibiera el patrocinio de la misma, muchos clubs tendrían que buscar nuevos sectores para publicitar sus clubs. 
Para terminar este bloque tres con la figura 14, los patrocinadores más locales se ha comprobado que son más utilizados por los clubs de hockey y en cambio los patrocinadores más nacionales e internacionales para el futbol. Esto es debido a que las competiciones europeas de futbol y el balonmano y el seguimiento de público, sean un punto de inflexión importante para negociar patrocinadores más internacionales y nacionales.

\section{Conclusiones}

En este estudio se ha podido observar que en función de los diferentes deportes la situación del patrocinio es diferente. La situación actual por lo que hace referencia al número de licencias pero sobretodo en el número potencial de seguidores y su consecuente interés por las diferentes marcas comerciales resulta determinante. A pesar de ello es difícil establecer reglas fijas para entender la dinámica de cada deporte y mucho más la tendencia que pueden seguir en un futuro.

Así por ejemplo, los naming right se han consolidado más en el baloncesto y en el balonmano ofreciendo nombre de empresas en los nombres de sus clubs. En función del deporte hay distintas diversificaciones, es decir, distintos cambios referentes a la visibilidad, a las categorías de patrocinio y a los sectores empresariales. Por ello, será muy importante conocer bien la situación del club.

La visibilidad en la gran mayoría de clubs es bastante evidente. En las páginas web de los equipos de la máxima categoría deberían hacer una reestructuración en sus páginas web para acceder con más facilidad a sus patrocinadores. Ante una situación más competitiva en los diferentes sectores, las empresas que patrocinan serán más exigentes en el retorno de sus inversiones.

Los dominios han servido para analizar la línea estratégica que pueden tener a nivel de marketing. En función de la propiedad online que se tenga se conocerá que patrocinadores buscarán los distintos clubs o incluso el tamaño de las empresas. Un club que obtenga un dominio «.cat» no buscará habitualmente patrocinadores multinacionales sino que serán de un carácter más local o regional. Por el contrario un «.com», buscará o tendrá un carácter más internacional.

La media de patrocinadores nos ha ayudado a ver las diferencias que existen en los deportes. Se ha examinado que la media de sponsors en el baloncesto ha sido 38, en el balonmano 24, en el futbol 14 y 13 en el hockey. Al tener menos patrocinadores se le da más exclusividad y más protagonismo que a la vez se verá recompensada con la visibilidad y la fuente de ingresos. La jerarquización marcada por las categorías de patrocinio ayudará a situar a los patrocinadores en diferentes líneas de negocio. Hay que tener en cuenta que es mejor para los clubs, si tener muchos patrocinadores y tener muchas fuentes de ingresos diferenciadas o si por el contrario, es mejor tener menos sponsors pero más potentes a nivel económico.

Los sectores empresariales deberían ampliarse más, para así abarcar mejor los patrocinadores en los distintos deportes. Los sectores más potentes son el alimentario, el financiero y el textil, representando a las grandes empresas de cerveza, los bancos que financian a los clubs y a los sponsors técnicos (marcas de ropa) de todos los equipos.

Finalmente, este estudio ha sido un primer paso en el desarrollo de la metodología de análisis del patrocinio. La falta de recursos no ha permitido estudiar los ingresos por patrocinio a nivel económico. En un futuro, la ficha que se ha creado para recoger la información de los clubs, podrá servir para recoger otras variables de otros deportes u organizaciones y así ampliar y profundizar más en otros estudios más globales de patrocinio deportivo.

\section{Referencias}

Acuña, A. \& Acuña, G. (2018). Valores del espectáculo de fútbol en el estadio: un estudio de caso. Retos, 33, 96-101.

Alonso-Dos-Santos, M., Calabuig, F., Montoro, F., \& Valantine, I. (2017). Influence of CSR in the image transmission in the sports sponsorship tourism. Revista Iberoamericana De Psicologia Del
Ejercicio YEl Deporte, 12(1), 23-31.

Antoine, C. (2007). Patrocinio y esponsoring en el deporte. La comunicación por el acontecimiento. Revista RE-Presentaciones, 3, 167183.

Bello, L. (1989). Esponsorización y mecenazgo en la estrategia de comunicación empresarial. Economía Industrial, 137-147.

Calderón, A., Nicolau, J. L., \& Mas, F. J. (2003). Factores determinantes de los resultados derivados de las actividades de patrocinio y mecenazgo, 24.

Campos, C. (1997). Marketing y Patrocinio Deportivo. (G. C. G. Deportiva, Ed.). Cáceres (España): Universidad de Extremadura.

Carroggio, M. (1994). Informe sobre el patrocinio deportivo en España. Barcelona: Universitat Autònoma de Barcelona (Barcelona).

Davis, K. A. (1994). Sport Management. Successful Private Sector Business Strategies. WCB \& Ben Chmark.

Desbordes, M., Ohl, F. \& Tribou, G. (2001). Estrategias del marketing deportivo. Paidotribo.

Diccionario Real Academia Española. (2001). Diccionario de la Lengua Española (VigésimaS).

Femenía, S. (2012). La Gestión del Patrocinio Múltiple en el Ámbito Deportivo: Perspectiva Relacional yde Red. Universidad Cardenal Herrera-CEU.

Gómez, O. (1993). Antecedentes y causas del desarrollo de la esponsorización y el patrocinio deportivos. Revista Apunts. Educación Fisica Y Deportes, 64-73.

Gómez, O. (2001). Comportamiento empresarial en materia de patrocinioy esponsorización deportiva. Revista Apunts. Educación Fisica Y Deportes, 64-75.

Hàbits Esportius a Barcelona-2013.(2014). Registre Públic d'Enquestes I Estudis D'opinió, 216-217.

Leruite, M. T., Cabrera, P. \& Zabala, M. (2015). Análisis del deporte femenino español de competición desde la perspectiva de protaginistas clave. Retos, 28, 3-8.

Maragas, M., Carroggio, M., Jones, D., Gutiérrez, M. \& Garcia, E. (2003). Patrocinio, comunicación y deporte (2nd ed.). Madrid: Ministerio de Educació y CSD.

Nogales, J. F. (2006). Uso y gestión del patrocinio deportivo: El patrocinio del balonmano, 2, 37-44.

Pérez-González, B., García-Unanue, J., Sánchez-Sánchez, J., SánchezBurón, A. \& Burillo, P. (2018). Análisis del comportamiento psicoeconómico del consumidor habitual de productos deportivos. Retos, 33, 267-272.

Sanajahu, G., Campos, C., Breva, E. \& Mut, M. (2015). Primer estudio sobre el estado del patrocinio deportivo en españa.

Slack, T., \& Barret, T. (1999). Corporate sponsorship and organisational strategy: Bridging the gap. International Journal of Sports Marketing \& Sponsorship, 1, 261-277.

Uefa. (2015). The European Club Footballing Landscape. Europe, 5763.

Urriolagoitia, L. E. (2007). El ciclo de la vida de las relaciones de patrocinio: Desarrollo de un modelo desde la perspectiva de las alianzas estratégicas. TDX (Tesis Doctorals En Xarxa). Retrieved from http:/ /www.tdx.cat/handle/10803/9191

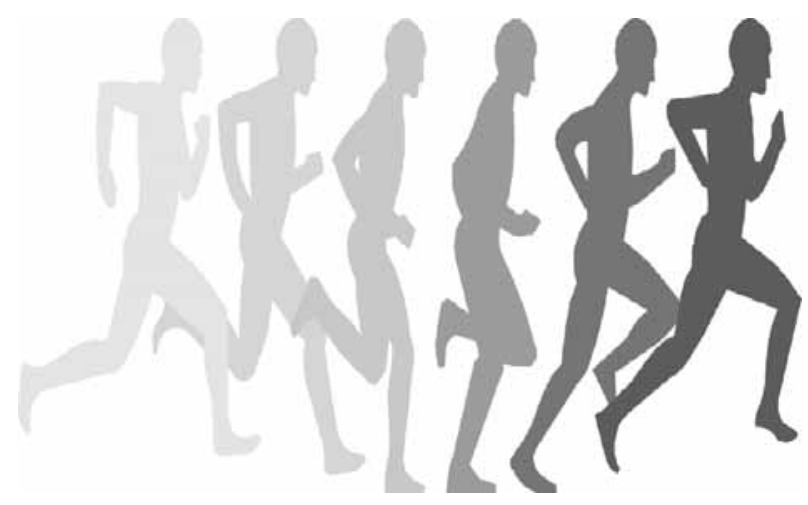

